\title{
INFORMATION ABOUT DYNAMICS OF THE SEA SURFACE AS A MEANS TO IMPROVE SAFETY OF THE UNMANNED VESSEL AT SEA
}

Marek Przyborski, Prof.

Gdansk University of Technology, Poland

\begin{abstract}
One of the fundamental states of the sea surface is its heave. Despite of years of the intense scientific inquiry, no clear understanding of the influence of this aspect on the dynamics of the sea environment has emerged. The separation of two nearby fluid elements which one may observed for example as a free floating of small objects on the sea surface (rescuers on the rough sea or small research vessels) is caused by the interaction of different components. On the other hand one may say that the heave of the sea is also a summary interaction of a few components describing the dynamics of the sea. Therefore it is the most important aspect, which influenced the dispersion phenomenon. This observation has important consequences for many different problems as for example conducting Search and Rescue missions and using unmanned ships. We would like to present results of our experiment focused on finding the answer to question about nature of the heave of the sea and its influence on safety of Unmanned Surface Vessels (USV).
\end{abstract}

Keywords: hypothesis testing, nonlinearity, sea dynamics, USV

\section{INTRODUCTION}

Heave of the sea is one of the most important factors that influenced the ship's movement especially at the open sea but also at the shallow waters. We may see its influence on the behavior of small boats, research vessels and unmanned surface vessels doing bathymetric tasks, laser scanning of the cliffs or infrastructure of harbor facilities $[7,9,11,14,17,18]$. The mathematical description of that phenomenon gives us the possibility to improve the quality of the data [22] dedicated to the spatial information systems and electronical navigational charts $[16,20]$ integrating information about shore infrastructure, shape of the sea bottom and waterways in the vicinity of the ports $[12,13,15,21]$.

One may say that it is the fusion of different components like sea currents, wind, atmospheric pressure, salinity, water temperature and so on. It influenced such a complex phenomenon like dispersion of small objects on the high seas. If this kind of behavior has a deterministic component then we may use deterministic methods like time series analysis to investigate its influenced on process of small objects dispersion. If it is not true then the heave of the sea might be considered simply as a disturbance, which influenced dispersion as a stochastic component.

Measuring of the heave of the sea is very difficult there are no clear and simple recording methods of this phenomenon. By using semiconductor accelerometer we have attempt a trial of collecting some measurements describing different states of natural sea environment. As a result of our experiment we 
have obtained a collection of time series of acceleration in three dimensions. When looking at this time series immediately one critical question has emerged - are there any deterministic components? If the distribution of this accelerations is totally random then heave of the sea should has also stochastic component and all the attempts to investigate this phenomenon by using deterministic methods must fail due to the stochastic nature of this process. If otherwise, searching for deterministic signatures we can answer the question about the nature of the heave of the sea and then, this would substantiate using nonlinear time series analysis methods to investigate such important phenomenon. So far many attempts have been made to detect behavior characteristic for deterministic systems in the data coming from the real world. Finite number of points as well as finite resolution of those kind of data makes this investigation difficult to obtain satisfactory results. In this particular case we would like to use the method of surrogate data $[5,6,19]$, which provides statistical test for the null hypothesis that the data has been generated by a linear stochastic process. If this null hypothesis cannot be rejected, then results of using nonlinear methods of analysis are not correct. This kind of test is based on comparing the value of nonlinear measure for the original data and a number of surrogates.

In this paper we will present results of applying such a test to seek for signatures of determinism in the samples of accelerations measured at high seas by the accelerometer mounted on small drifter.

\section{EXPERIMENT DESCRIPTION}

The null hypothesis, which is suitable for our investigation, states that the interesting us data was generated by a stationary Gaussian linear stochastic process. Computing nonlinear observable on the data allows distinguishing deviations from the null hypothesis, our main purpose is to show that the original data $x_{n}^{0}$, differs significantly from the surrogates which are realizations of the null hypothesis. Our original data consists of samples of accelerations recorded in 3-dimensions

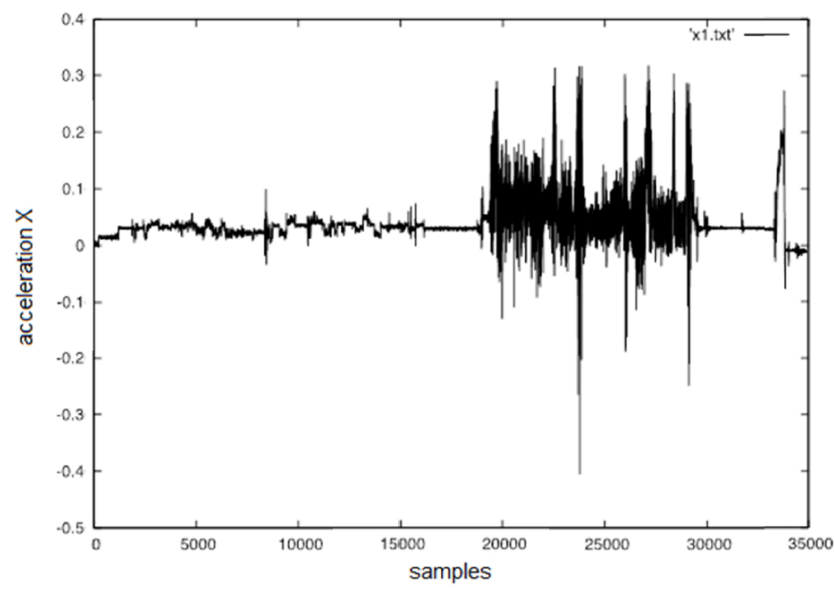

Fig. 1. Acceleration in $X$ direction

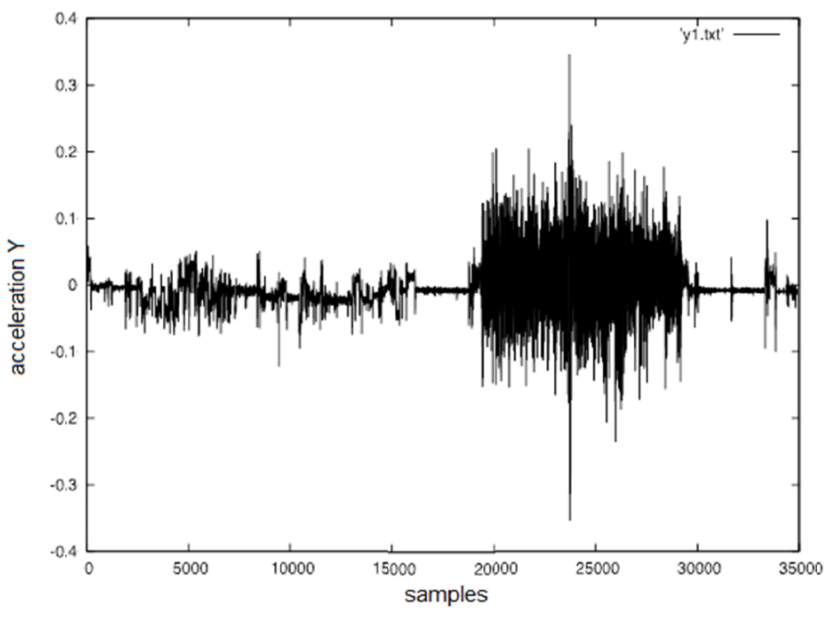

Fig. 2. Acceleration in Y direction

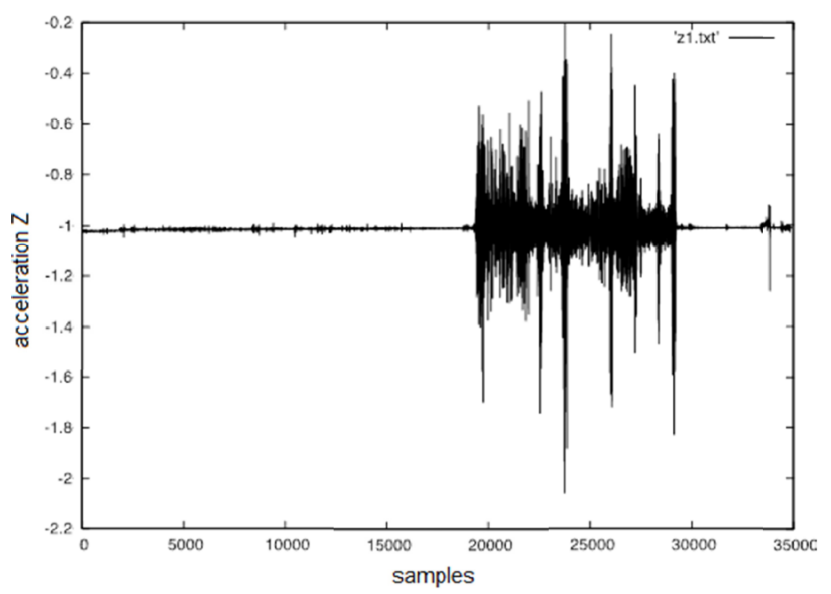

Fig. 3. Acceleration in $Z$ direction

while the drifter was moving on the sea surface. We have used system of drifters equipped with GPS and radio link with the main station on board the research vessel. This technique has been used for the first time for tracking the position of drifters in order to find the distribution of the sea currents. The laboratory equipment consist of 5 drifters with mass about $80 \mathrm{~kg}$ each one. We may assume that the dimensions of the drifters are small comparable to the open sea. During our previous experiments we have come to the conclusion that the distribution of the sea currents in the Baltic Sea is extremely difficult to describe. $w$. The heave of the sea is one of the most important factors when considering dynamics of the sea environment. By recording the accelerations we tried to described the specific state of the interested us environment. When talking about the analysis, first we have to answer the question about the nature of collected data. Is it deterministic or not? If yes, then we may use all the methods coming from the time series analysis in particular some methods form deterministic chaos. In our search for nonlinearity in those data we used method of surrogate date.

The surrogate data is a set of data which mimic the original one, however consistent with the null hypothesis. For a given conjecture we generated $\left\{x_{n}^{k}\right\}, k=1, \ldots, B$ surrogates of the 
specific type, in our case they should be realization of the Gaussian linear stochastic process.

As an example of the data, Fig. 1, 2, 3 presents the accelerations recordings, this particular segment comes from the first sea trial. The Fig. 4 presents an example of surrogate data created to mimic accelerations in $\mathrm{X}$ direction.

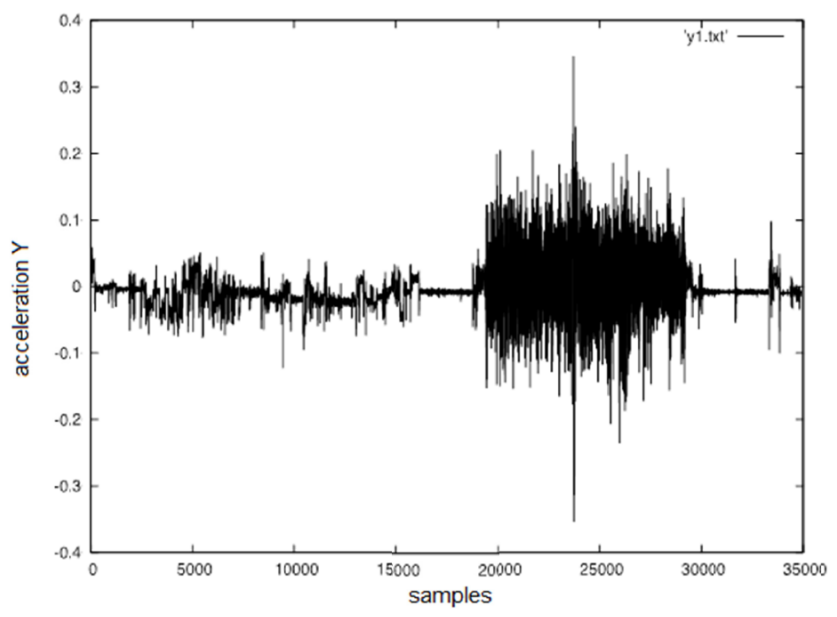

Fig. 4. Surrogates of the data presented on the Fig. 1

When creating tests for nonlinearity we should take into account two parameters. The first one, is its size $\alpha$, which is the probability that the null hypothesis is rejected although it is in fact true. And the second, called level of significance $1-p$ (usually the value of $p$ is specify a priori, and then test is design with accordance to its value).

\section{TESTING FOR NONLINEARITY WITH SURROGATE DATA}

The null hypothesis which is suitable for our investigation states that the data was generated by a stationary Gaussian linear stochastic process. Computing nonlinear observable on the data allows distinguishing deviations from the null hypothesis, our main purpose is to show that the original data $x_{n}^{0}$, differs significantly from the surrogates which are realizations of the null hypothesis. Our original data (Fig. $1,2,3)$ consists of samples of accelerations measured on the high seas. Recordings were done on the standard PC computer equipped with the RS-232 card and downloaded into the hard disk as a .txt files. We have collected recordings from 4 sea trails. Each one of them consists of about 300.000 samples. Due to the length of the recording, we split it, and for further considerations we have used only segments of length 10000 points. We decided to choose this length, due to stationarity requirement.

\section{MEASURES OF NONLINEARITY}

There exists several examples of different statistical methods (see for instance Ref. $[1,2,6]$ ) which have been developed in order to reveal the nature of the considered time series.
We decided to apply two types of nonlinear statistics $t=t\left(\left\{x_{n}\right\}\right)$ :

1. As a first one, nonlinear prediction error with respect to a locally constant predictor $F$ defined by

$$
t^{1}(m, \tau, \epsilon)=\left(\sum\left[x_{n+1}-F\left(x_{n}\right)\right]^{2}\right)^{1 / 2}
$$

The prediction is performed over one time step and it is done by averaging over the future values of all neighboring delay vectors closer than $\epsilon$ in $m$ dimensions.

2. The second quantity it is cross-prediction errors, which can be expressed by the following formula

$$
\sigma_{X, Y}^{2}=\frac{1}{L^{\prime}} \sum_{k=(m-1) \tau+1}^{L-1}\left\|\vec{y}_{k+1}-F_{X}\left(\vec{y}_{k}\right)\right\|^{2}
$$

where: $L^{\prime}=L-((m-1) \tau+1)$ - number of delay vectors, and $F$ zero-th order model as it is proposed in the Ref. 7].

Calculating those nonlinear observable require using time delay embedding according to the following scheme, where embedding vectors in $m$ dimensions are created by: $\vec{x}_{n}=\left(x_{n-(m-1) \tau}, \ldots, x_{n}\right),-\tau$ is the delay time.

\section{RESULTS}

We generated the surrogate data according to the null hypothesis which states that a Gaussian linear stochastic process has generated the data. This is the simplest description of a purely stochastic process, therefore we decided to applied this hypothesis. The method is based on the phase randomized surrogate series $S=\left\{s_{n}, n=1, \ldots, N\right\}$ which has the same power spectrum as the time series $X=\left\{x_{n}, n=1, \ldots, N\right\}$. The temporal correlations in the original data are not preserved in the surrogates. The surrogate is obtained by determine the Fourier transform of the original data $X$, randomizing the phases, and inverting the transform. The probability distributions of the nonlinear statistic $t=t\left(\left\{x_{n}\right\}\right)$ remains unknown to us, therefore we applied a non-parametric, rankbased test. If the data deviates from the surrogates in a specific direction then we can reject the null hypothesis (one-side test). According to the size of the test $\alpha$, we create $B=1 / \alpha-1$ surrogate sets and then compute the test statistic $t_{0}$ on the original data and on each of the surrogates $\left(t_{k}, k=1, \ldots, B\right)$. For the prediction error, we expect nonlinearity in the data to appear in the lower values. Thus in this case we perform one-sided tests. All tests were carried out at the 95\% level of significance, it means that for one-sided test we have created 19 surrogates. Our data is a typical example of field measurement, it is strongly contaminated by the noise. In this particular case the nature of noise can have dual substance, the first source is a measurement noise, and the second one as we have already said is the influence of for example wind.

Assuming that the second type of noise and the samples of accelerations can be well described by the Gaussian linear 
stochastic process [3] we can expect that those features should be reflected in the results of the test. Simply in the presence of the stochastic components in our data test for nonlinearity should fail. Conducted tests reveal that for one-sided test we achieved the 95\% level of significance each time, results of one of the tests are presented on the Fig. 5.

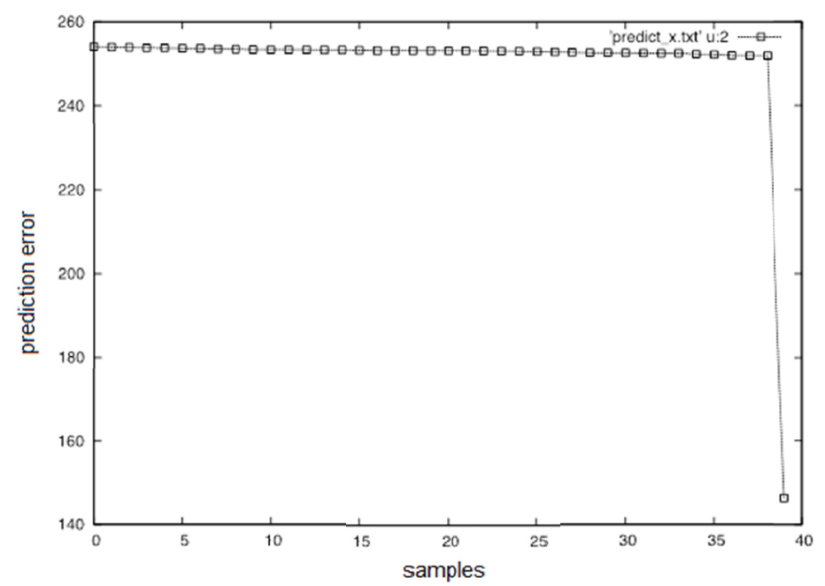

Fig. 5. Prediction error for the series of accelerations in $X$ direction.

In order to precise our results we decided to apply method based on the clustering algorithm. The main idea of using this algorithm in the test for nonlinearity is to classify the total set of surrogates and original data, say $K$ into two groups, from which one has only 1 element. As a dissimilarity measure cross-prediction errors were used. For conducting the test we used 9 surrogates and the original data, thus the probability that if the algorithm turned out the original data is $1 / K=$ 0.1 , if it is true then we can reject the null hypothesis with the $(1-(1 / K)) \times 100=90 \%$ of significance. For each combination of surrogates and original data from the set of 30 segments we obtained the rejection of the null hypothesis with the $90 \%$ level of confidence. An example of the answer given by the clustering algorithm is presented on the Fig. 6 .

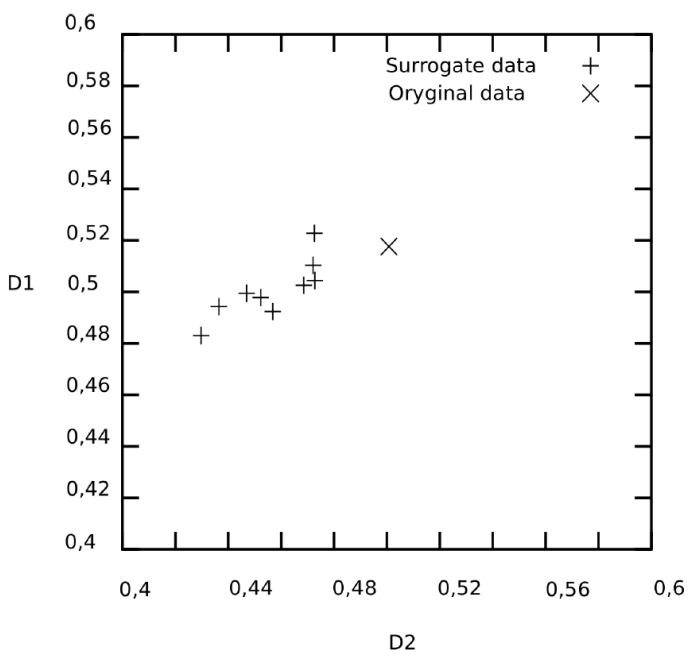

Figure 6. Results of clustering algorithm
As we have seen there are two clusters and one of them singled out the one element, which contain the original data.

\section{CONCLUSION}

We applied two nonlinear statistics in order to reveal the possible nature of considered signals. Taking into account results of conducted tests we can conclude that the null hypothesis can be rejected because original data cannot be well described by the Gaussian linear stochastic process. It means that our assumption about the nature of samples of accelerations influenced the move of experimental drifter is false, thus its distribution can be predicted due to deterministic nature of that process.

The null hypothesis, which has been chosen, in fact is the simplest one, however in our opinion it presents the main drawback in the field of investigation such a complicated phenomenon like dynamics of the sea environment. The possibility to reject the hypothesis of stochastic nature of that signals opens the way to extract the most important features of the considered object by means, for example nonlinear time series analysis. This will allow us to characterize sea surface dynamics by the set of suitable parameters.

\section{ACKNOWLEDGEMENT}

Publication of the above text and its language translation was funded by the Ministry of National Education of the Republic of Poland - the task BGC Innovation Forum Surveying in Geodesy, the contract 965 / P-DUN / 2016, allocated to the activities of disseminating science.

\section{REFERENCES}

1. Diks, C., et al. Reversibility as a criterion for discriminating time series. Phys. Lett. A. 1995, p. 201:221, DOI: 10.1016/0375-9601(95)00239-Y.

2. Kantz, H. and Schreiber, T. Nonlinear time series analysis. Oxford : Cambridge University Press, 1997.

3. Percival, D. B. and Walden, A. T. Spectral analysis for physical applications. Cambridge : Cambridge University Press, 1993.

4. Thailer, J., et al. Nonlinear modeling and forecasting. Using surrogate data to detect nonlinearity in time series. Reading : Santa Fe Institute Studies in the Science of Complexity,. Vol. Proc. Vol. XII, 1992

5. Schreiber, T. Constrained randomization of time series data. Phys. Rev Lett., Vol. 80, 1998, DOI: 10.1103/ PhysRevLett.80.2105

6. Theiler, J. and Prichard, D. Generating surrogate data for time series with several simultaneously measured variables. 
Phys. Rev. Lett., Vol. 73, 1994

7. Burdziakowski, P. et al. Maritime laser scanning as the source for spatial data. Polish Maritime Research, Vol. 22, Iss. 4, pp. 9-14, 2015, DOI: 10.1515/pomr-2015-0064

8. Hejmanowska, B. et al. Modern remote sensing and the challenges facing education systems in terms of its teaching. 7th International Conference on Education and New Learning Technologies, Barcelona, Spain JUL 06-08, 2015, pp. 6549-6558, 2015

9. Janowski, A. et al. Airborne and mobile laser scanning in measurements of sea cliffs on the southern Baltic. 15th International Multidisciplinary Scientific Geoconference (SGEM) Location: Albena, Bulgaria, JUN 18-24, 2015, SGEM 2015, pp. 17-24, 2015, DOI: 10.5593/SGEM2015/ $\mathrm{B} 12 / \mathrm{S} 2.003$

10. Janowski, A. et al. Proprietary software in technical higher education. EDULEARN14: 6th International Conference On Education and New Learning Technologies, ISBN 978 84-617-0557-3, ISSN 2340-1117, pp. 1941-1949, 2014

11. Jedrasik J. and Szymelfenig M.: Ecohydrodynamic model of the Baltic Sea. Part 2. Validation of the model, Oceanologia, Vol. 47, Iss. 4, pp. 543-566, 2005

12. Kazimierski, W. and Stateczny, A. Radar and Automatic Identification System Track Fusion in an Electronic Chart Display and Information System. Journal of Navigation, Vol. 68, Iss. 6, pp. 1141-1154, 2015, DOI: 10.1017/ S0373463315000405P

13. Kazimierski, W. et al. Verification of multiple model neural tracking filter with ship's radar. 13th International Radar Symposium (IRS) Location: Warsaw, Poland, MAY 23-25, 2012, Book Series: International Radar Symposium Proceedings, pp. 549-553, 2012, DOI: 10.1109/ IRS.2012.6233384

14. Kedzierski, M. et al. Detection of gross errors in the elements of exterior orientation of low-cost UAV images. Baltic Geodetic Congress (Geomatics), Gdansk, Poland, JUN 2-4, 2016, pp. 95-100, 2016, DOI: 10.1109/BGC. Geomatics.2016.26

15. Kozaczka, E. et al. Detection of Objects Buried in the Sea Bottom with the Use of Parametric Echosounder. Archives of Acoustics, Vol. 38, Iss. 1, pp. 99-104, 2013

16. Kulawiak M., Chybicki A., Moszynski M.: Web-based GIS as a tool for supporting marine research. Marine Geodesy, Vol. 33, Iss. 2-3, pp. 135-153, Art. No. PII 923578807, 2010, DOI: $10.1080 / 01490419.2010 .492280$

17. Liao Yu-lei, Su Yu-min, Cao Jian. Trajectory planning and tracking control for under actuated unmanned surface vessels. Journal of Central South University, Vol. 21, Iss. 2, pp. 540-549, 2014, DOI: 10.1007/s11771-014-1972-x

18. Michoud, C. et al. Landslide detection and monitoring capability of boatbased mobile laser scanning along Dieppe coastal cliffs, Normandy. Landslides, Vol. 12, Iss. 2, pp. 403-418, 2014, DOI: 10.1007/s10346-014-0542-5

19. Przyborski, M. Possible determinism and the real world data. Physica A-Statistical Mechanics and Its Applications, Vol. 309 Iss. 3-4, pp. 297-303, PII S0378-4371(02)00565-4, 2002

20. Roberts, G. N. Trends in marine control systems. Annual Reviews in Control, Vol. 32, Iss. 2, pp. : 263-269, 2008, DOI: 10.1016/j.arcontrol.2008.08.002

21. Stateczny, A. and Bodus-Olkowska, I. Hierarchical Hydrographic Data Fusion for Precise Port Electronic Navigational Chart Production. 14th Transport Systems Telematics Conference (TST) Location: Poland Date: OCT 22-25, 2014, Book Series: Communications in Computer and Information Science, Vol. 471, pp. 359-368, 2014, DOI: 10.1007/978-3-662-45317-9_38

22. Stateczny, A. and Bodus-Olkowska, I. Sensor data fusion techniques for environment modelling. 16th International Radar Symposium (IRS) Location: Dresden, Germany, JUN 24-26, 2015, Book Series: International Radar Symposium Proceedings, pp. 1123-1128, 2015, DOI: 10.1109/IRS.2015.7226263

\section{CONTACT WITH THE AUTHOR}

\author{
Marek Przyborski \\ e-mail: marek.przyborski@pg.gda.pl
}

Gdańsk University of Technology

Department of Geodesy

Faculty of Civil and Environmental Engineering 11/12 Narutowicza St. 80 - 233 Gdańsk

Poland 